\title{
Boundary Avoidance Tracking for Instigating Pilot Induced Oscillations
}

\author{
Robert W. Craun ${ }^{1}$ \\ Mission Critical Technologies, Inc., Moffett Field, California, 94035 \\ Diana M. Acosta ${ }^{2}$ and Steven D. Beard ${ }^{3}$ \\ NASA Ames Research Center, Moffett Field, California, 94035 \\ and \\ Gordon H. Hardy ${ }^{4}$, Michael W. Leonard ${ }^{5}$, and Michael Weinstein ${ }^{6}$ \\ Science Applications International Corporation, Moffett Field, California, 94035
}

\begin{abstract}
In order to advance research in the area of pilot induced oscillations, a reliable method to create PIOs in a simulated environment is necessary. Using a boundary avoidance tracking task, researchers performing an evaluation of control systems were able to create PIO events in $42 \%$ of cases using a nominal aircraft, and $91 \%$ of cases using an aircraft with reduced actuator rate limits. The simulator evaluation took place in the NASA Ames Vertical Motion Simulator, a high-fidelity motion-based simulation facility.
\end{abstract}

\section{Introduction}

$\mathrm{F}$ $\mathrm{OR}$ as long as aircraft have flown, pilot induced oscillations have presented a significant problem for both pilots and engineers. Many pilots have experienced at least a minor pilot induced oscillation (PIO). Major, divergent oscillations are rare, but these PIOs can be catastrophic. While practical design considerations and analytical methods have been developed for PIO prevention, PIO events continue to occur, especially during the initial flight-testing of aircraft. Many design choices that enhance an aircraft's energy efficiency make the aircraft susceptible to PIOs. To allow future aircraft to meet improved efficiency goals, research is needed to mitigate the effects of PIO factors and PIO events.

To investigate methods to prevent or recover from PIOs, PIOs must be created consistently, in large numbers, under controlled circumstances, and cheaply. Testing in real aircraft can be hazardous, unpredictable, and costly. Creating PIOs in simulators has not proven effective due to the lower pilot gain associated with not having real hazards and reduced motion cues. These circumstances have presented a challenge for engineers researching methods to prevent and recover from PIOs. In 2004, William Gray developed the concept of Boundary Avoidance Tracking (BAT), which puts the pilot between two opposing boundaries, and devised a task to create PIOs using BAT. ${ }^{4}$ It was implemented in simulators of increasing fidelity, and finally implemented in T-38s. It was shown that the BAT task had potential to reliably create PIOs in a consistent manner.

1 Former Aerospace Engineer, MCT, Inc, NASA Ames Research Center, Mail Stop 269-1. Currently Second Lieutenant in the United States Marine Corps.

2 Aerospace Engineer, Intelligent Systems Division, Mail Stop 269-1.

3 Simulation Engineer, Aerospace Simulation Research and Development Branch, Mail Stop 243-1, AIAA Senior Member.

4 Senior System Analyst, SAIC, NASA Ames Research Center, Mail Stop 243-6.

5 Former Senior Simulation Engineer, SAIC, NASA Ames Research Center, Mail Stop 243-6. Currently Senior Member of Consulting Staff, Mentor Graphics, Inc., 46871 Bayside Parkway, Fremont, California, 94538.

6 Senior Simulation Engineer, SAIC, NASA Ames Research Center, Mail Stop 243-6. 
When a simulation experiment was under development to evaluate the effectiveness of different control systems in recovering from PIOs, a method to create PIOs was needed. A BAT task was utilized to create PIOs in a controlled, repeatable manner to meet experiment objectives. The implementation of the BAT task was defined by two pairs of magenta needles arranged similar to a tic-tac-toe board on the Primary Flight Display (PFD), as shown in Figure 1. The pilot was required to keep the bore-sight of the aircraft within the center box as long as possible as it moved vertically to a sum-of-sines pattern. The horizontal magenta lines became progressively closer as the task went on. There were four configurations used for the control system experiment, two of which had actuator rate limits of $100 \% \mathrm{~s}$ and two had actuator rate limits of $40 \% \mathrm{~s}$. A total of seven pilots performed 121 data runs.

This paper provides a background on PIOs in Section II, describes the PIO simulation study in Section III, and discusses the outcome of this BAT task implementation in Section IV, including the task's ability to generate PIOs and the correlation between PIO activity and task performance. Recommendations for future implementations of the BAT task in simulation are also provided.

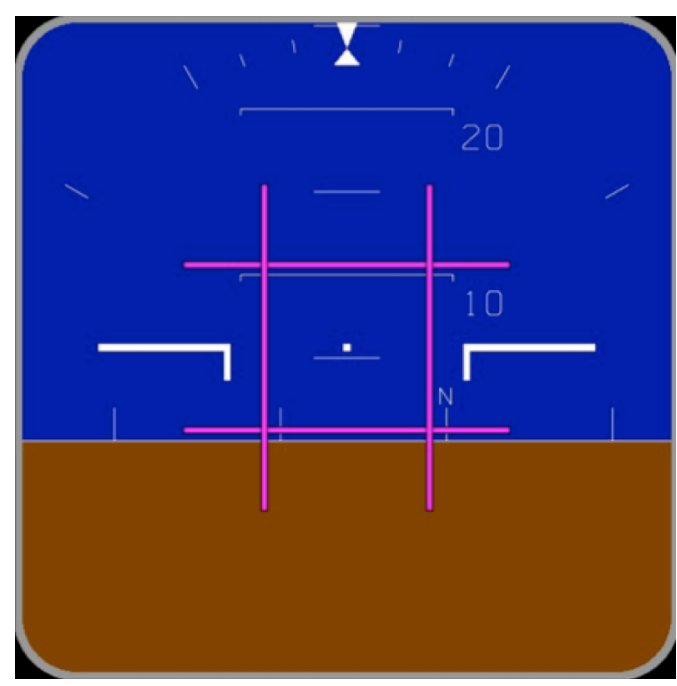

Figure 1. PFD displaying BAT task.

\section{Background}

\section{A. Pilot Induced Oscillations}

A Pilot Induced Oscillation (PIO) is a sustained or uncontrollable, inadvertent oscillation resulting from the pilot's efforts to control the aircraft. ${ }^{1}$ The pilot reacts to the motion of the aircraft, creating a closed-loop feedback control system. The oscillations can therefore be identified as closed-loop instabilities of a feedback control system. ${ }^{2}$

During a PIO, there are phase lags between the pilot's commands and the aircraft's responses. A typical PIO is characterized as "an oscillation at a frequency where the attitude response lags the stick inputs by approximately 180 degrees." ${ }^{3}$ The onset of some types of PIOs can be recognized in the conceptual diagram in Figure 2, which depicts the phase lag between a pilot's control signal and a rate-saturated control surface response. While PIOs can be easily identified during post-flight data analysis, often pilots do not know they are in a PIO-from their perspective the aircraft appears to have broken. ${ }^{3}$

\section{B. Contributing Factors to PIO}

Three contributors must be present to induce a PIO; these contributors are the aircraft, the pilot, and the trigger. The aircraft can contribute to a PIO by having lags in the control system, unstable or marginally stable modes, slow

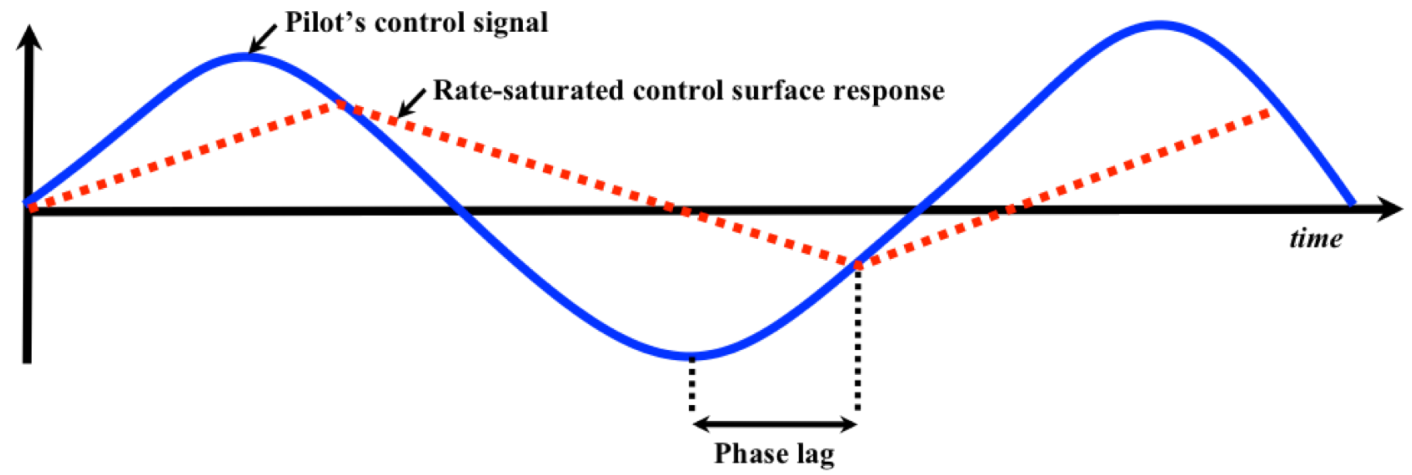

Figure 2. Diagram depicting phase lag between a pilot's control signal and a rate-saturated control surface response. 
actuators that cannot meet the demands of the pilot, or a combination of these factors. Pilots are the driving factor in a PIO, sustaining the oscillations by inputting higher than normal gain or leading signals to the control system. ${ }^{3}$ Finally, the piloted task or trigger is the impetus for increasing the pilot gain and starting the PIO. In PIO theory, the interplay between the aircraft, pilot and trigger has been broken down into two parts. One part is the interaction between the pilot and the trigger; the second part is between the pilot and the vehicle system. These interactions are important to understanding when an aircraft may be vulnerable to a PIO.

\section{Pilot-Trigger Interaction}

A pilot flies an aircraft using one of two tracking methods. The first, and most common, is point tracking. In point tracking tasks, the pilot is attempting to track or converge on a point, such as the probe of an aerial refueling boom or the wingtip of the leader in formation flight. Maintaining an assigned altitude is another example of a point tracking task. As the pilot closes in on the desired point, the distance and associated time constants decrease. This leads to increased pilot gain and increased frequency of stick inputs required to track the desired point, which can trigger a PIO. For instance, when the pilot overshoots the nominal point, the resultant correction coupled with any vehicle latencies, leads to an overshoot in the opposite direction and the start of a PIO. The pilot is usually trained to go "hands off" and leave the control loop, ending the PIO.

The second tracking method that can trigger a PIO is boundary avoidance tracking. This usually occurs during landing or low altitude flight, but can occur in any situation where the pilot is stuck between two opposing boundaries (e.g. G-load, angle of attack (AoA), pitch/roll limits, or physical boundaries). As an example, if a nosedown gust hits the aircraft as it crosses the runway threshold, the pilot, to avoid damage from a ground strike, will pull the aircraft sharply up. Any latency in the system can cause the pilot to over-control. In this case, the overcorrection will lead the aircraft to rapidly approach the upper AoA boundary, which can be catastrophic at low altitude. The pilot would then push forward on the stick to stop the aircraft's movement towards the critical AoA, sending the nose towards the runway, setting off a PIO. In this case the pilot is inclined to stay in the loop, since a PIO is initially a better option then exceeding either boundary.

\section{Pilot-Vehicle Interactions}

Pilot-vehicle interactions are described by three categories of PIOs. In Category I PIOs, the vehicle characteristics are essentially linear, and the pilot behaves in a quasi-linear manner. The oscillations are caused by high open-loop gain. Category I PIOs are more repeatable, can be easily backed out of by the pilot, and are the least threatening.

A quasi-linear aircraft with rate or position limiting characterizes Category II PIOs. Additionally, nonlinearities such as stick command shaping or aerodynamics properties may also exist in a Category II PIO. The rate limiting of actuators can turn Category I PIO into a Category II by adding lag when there are large commanded inputs.

Category III PIOs are the result of serious nonlinearities within the aircraft system, such as mode switching in the software, or sudden hardware or aerodynamic changes. Category III PIOs can also result from a pilot switching tracking behaviors or input cues. PIOs in this category are always severe.

\section{Development of the Boundary Avoidance Tracking Task}

William Gray of the USAF Test Pilot School created the theory of Boundary Avoidance Tracking (BAT) in 2004, offering an alternative to the point tracking theory of PIO formation. ${ }^{4}$ The first experiment exercising this theory as a pilot task was in 2005. A piloted simulation using the North American Navion's flight dynamics performed an altitude-tracking task with an upper and lower boundary. The boundaries were brought $25 \%$ closer every minute, and the oscillatory flight path of the leader repeated every minute. The task was over immediately after the pilot exceeded the boundaries, with the goal of staying inside as long as possible.,

Following this first experiment, a series of piloted experiments in T-38's took place at the USAF Test Pilot School. In these experiments, the lead aircraft flew a positive-G pull-up, a negative-G push-over, or combination, while the test subject was required to maintain formation without exceeding lines painted on the side of the lead aircraft. ${ }^{7}$ The BAT concept was further characterized by simulation and flight studies using the Infinity Cube simulator, the Large Amplitude Multi-Mode Aerospace Research Simulator (LAMARS), and the NF-16D VISTA Test Aircraft. ${ }^{8}$

\section{Simulation Evaluation Design}

The objective of the PIO simulation study was to evaluate the effectiveness of different control systems in recovering from PIOs. In support of this objective, the simulation evaluation was designed to reliably create PIOs in a controlled, repeatable manner. This required a demanding task, realistic motion cues for the pilots, and extreme 


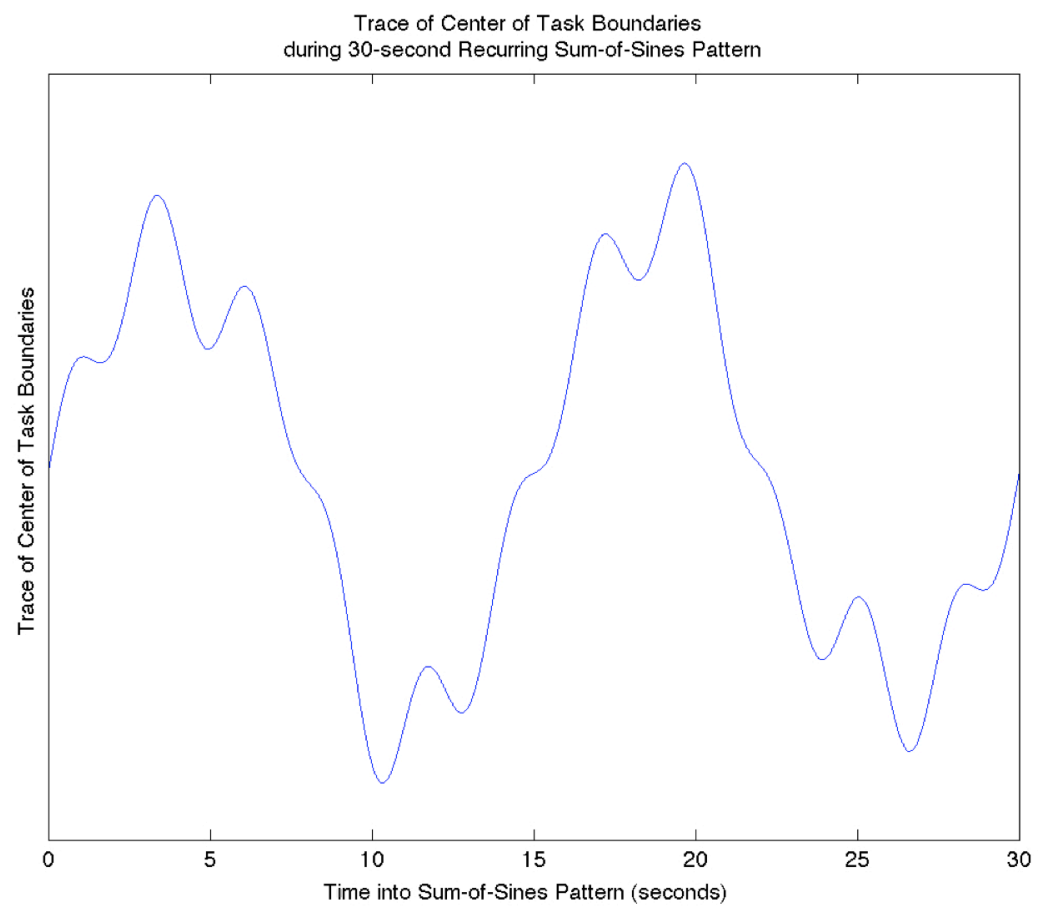

Figure 3. Trace of Center of Task Boundaries during Recurring Sum-of-Sines Pattern.

research aircraft configurations. Details on these attributes, as well as the experimental procedure and data collected, are described below.

\section{A. Boundary Avoidance Tracking Task}

The BAT task used during the simulation evaluation was developed based on previous BAT experiments described in Section II.C. The BAT task was designed to become progressively harder and expose PIO tendencies in the longitudinal axis as the pilot's control gain increased with the task difficulty.

The task was defined by two pairs of magenta needles arranged similar to a tic-tac-toe board on the Primary Flight Display (PFD), which are shown in Figure 1. The needles formed a box, and the pilot was required to keep the bore-sight of the aircraft within this box as the box moved up and down. The goal was to keep the bore-sight within the box as long as possible, and the task was terminated when the bore-sight encountered the box's boundary. The task started with the aircraft in straight and level flight, but since the box was continuously moving and the atmospheric condition included light turbulence, the task required pilots to stay tightly in the loop to avoid the boundaries. Pilots were required to focus exclusively on the box boundaries since there was no single point tracking element.

The vertical motion of the center of the box followed a sum-of-sines pattern, which repeated itself every 30 seconds. As the pattern repeated, the distance between the horizontal lines shrunk by $25 \%$. The parameters used for the sum-of-sines pattern are given in Table 1, and a trace of the center of the task boundaries during the recurring pattern is shown in Figure 3. The frequencies used are from Ref. 8; they were chosen because the resulting pattern has a 30 second period, allowing the whole pattern to repeat at the same time as the boundaries close in. The amplitudes were selected using trial and error to arrive at a pattern that was difficult yet achievable, and forced the pilots to stay active throughout the entire task. The amplitude values provided in Table 1 are scaled to conceal details of the research aircraft model. In order to minimize the chance that a pilot will memorize the pattern of the sum-of-sines, a variation was used that multiplied the sum-of-sines output by negative one. 
In an attempt to increase pilot gain, an audible tone played over the headsets as the bore-sight approached a boundary, warning of the impeding excursion. The frequency of the tone would increase as the distance to the boundary decreased. The tone augmented the visual boundary as an audio trigger to create a sense of urgency. After the bore-sight exceeded the boundary, a different, loud alarm played. This served to notify the pilot of the failure. In addition to the audible cues, a yellow warning light engaged with the warning tone to create a sense of urgency, and a red failure light turned on when the boundary was exceeded to notify the pilot of the failure. Both of these lights were located on the panel above the PFD.

\section{B. Vertical Motion Simulator Facility}

The Vertical Motion Simulator (VMS) is the ideal facility to expose PIO tendencies and test a control system's effectiveness in recovering from PIOs because of the VMS's large motion envelope. Schroeder, et. al. ${ }^{12}$ concluded that larger simulator motion envelopes provide more accurate HQR and PIO ratings than smaller simulator motion envelopes when compared to the same ratings taken in the actual aircraft. Schroeder also found that a large motion simulator was the only ground-based platform that induced markedly divergent PIOs. Additionally, pilots gave large motion higher confidence factor ratings and achieved lower touchdown velocities compared to small motion simulators.

The VMS motion system, shown in Figure 4, is an uncoupled, six-degree-of-freedom motion simulator. It is located in, and partially supported by, a specially constructed 120-ft tower. The VMS system motion capabilities are provided in Table 2. Included in the table are two sets of limits: system limits that represent the absolute maximum levels of attainable under controlled conditions; and operational limits, that represent attainable levels for normal piloted operations.

The cab, shown in white in Figure 4, serves as the aircraft cockpit. The evaluation pilot occupied the right seat, with the test engineer in the left. A computer image generation system creates the out-the-window visual scene for the six-window collimated display with the head-up display superimposed on the center window. Additional aircraft information was provided on three head-down displays at both pilot stations.

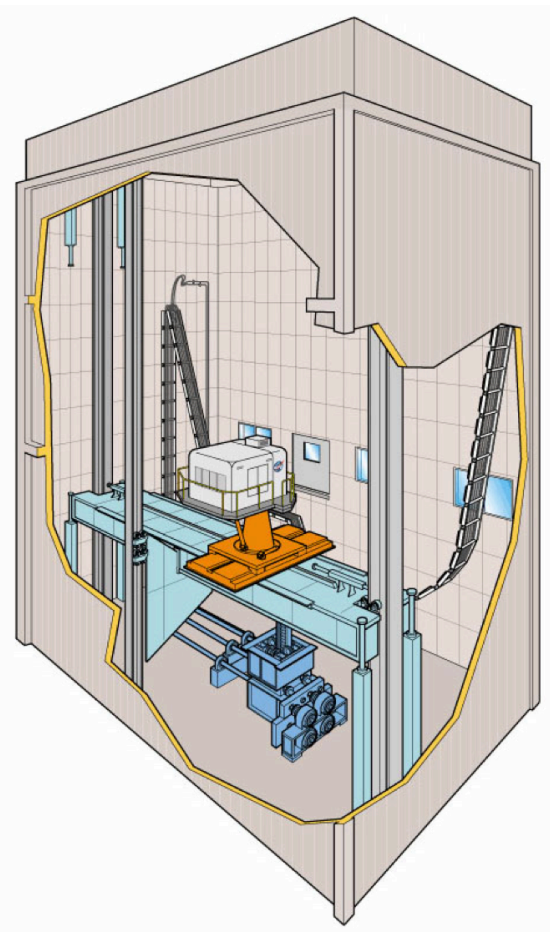

Figure 4. VMS facility.

Table 2. VMS Motion System Performance Limits. ${ }^{10}$

\begin{tabular}{|c|c|c|c|c|c|c|}
\hline \multirow{2}{*}{$\begin{array}{c}\text { Degree } \\
\text { of } \\
\text { Freedom }\end{array}$} & \multicolumn{2}{|c|}{ Displacement } & \multicolumn{2}{|c|}{ Velocity } & \multicolumn{2}{|c|}{ Acceleration } \\
\hline & $\begin{array}{l}\text { System } \\
\text { Limits }\end{array}$ & $\begin{array}{c}\text { Operational } \\
\text { Limits }\end{array}$ & $\begin{array}{l}\text { System } \\
\text { Limits }\end{array}$ & $\begin{array}{c}\text { Operational } \\
\text { Limits }\end{array}$ & $\begin{array}{l}\text { System } \\
\text { Limits }\end{array}$ & $\begin{array}{l}\text { Operationa } \\
\text { Limits }\end{array}$ \\
\hline Longitudinal & $\pm 4 \mathrm{ft}$ & $\pm 4 \mathrm{ft}$ & $\pm 5 \mathrm{ft} / \mathrm{sec}$ & $\pm 4 \mathrm{ft} / \mathrm{sec}$ & $\pm 16 \mathrm{ft} / \mathrm{sec}^{2}$ & $\pm 10 \mathrm{ft} / \mathrm{sec}^{2}$ \\
\hline Lateral & $\pm 20 \mathrm{ft}$ & $\pm 15 \mathrm{ft}$ & $\pm 8 \mathrm{ft} / \mathrm{sec}$ & $\pm 8 \mathrm{ft} / \mathrm{sec}$ & $\pm 13 \mathrm{ft} / \mathrm{sec}^{2}$ & $\pm 13 \mathrm{ft} / \mathrm{sec}^{2}$ \\
\hline Vertical & $\pm 30 \mathrm{ft}$ & $\pm 22 \mathrm{ft}$ & $\pm 16 \mathrm{ft} / \mathrm{sec}$ & $\pm 15 \mathrm{ft} / \mathrm{sec}$ & $\pm 22 \mathrm{ft} / \mathrm{sec}^{2}$ & $\pm 22 \mathrm{ft} / \mathrm{sec}^{2}$ \\
\hline Roll & $\pm 0.31 \mathrm{rad}$ & $\pm 0.24 \mathrm{rad}$ & $\pm 0.9 \mathrm{rad} / \mathrm{sec}$ & $\pm 0.7 \mathrm{rad} / \mathrm{sec}$ & $\pm 4 \mathrm{rad} / \mathrm{sec}^{2}$ & $\pm 2 \mathrm{rad} / \mathrm{sec}^{2}$ \\
\hline Pitch & $\pm 0.31 \mathrm{rad}$ & $\pm 0.24 \mathrm{rad}$ & $\pm 0.9 \mathrm{rad} / \mathrm{sec}$ & $\pm 0.7 \mathrm{rad} / \mathrm{sec}$ & $\pm 4 \mathrm{rad} / \mathrm{sec}^{2}$ & $\pm 2 \mathrm{rad} / \mathrm{sec}^{2}$ \\
\hline Yaw & $\pm 0.42 \mathrm{rad}$ & $\pm 0.24 \mathrm{rad}$ & $\pm 0.9 \mathrm{rad} / \mathrm{sec}$ & $\pm 0.8 \mathrm{rad} / \mathrm{sec}$ & $\pm 4 \mathrm{rad} / \mathrm{sec}^{2}$ & $\pm 2 \mathrm{rad} / \mathrm{sec}^{2}$ \\
\hline
\end{tabular}




\section{Research Aircraft and Flight Control System Configurations}

The research aircraft math model flown in this evaluation was the Speed Agile Concept Demonstrator (SACD) a short takeoff and landing mobility concept being developed by industry under the U.S. Air Force Research Laboratory's (AFRL) Advanced Joint Air Combat System studies. The SACD program seeks to mature technology in the areas of high lift, efficient transonic flight, and flight control for future integrated mobility configurations that are intended to carry larger, heavier payloads than the C-130, fly efficiently across a wide range of speeds, cruise above Mach 0.8 , and routinely operate from short, unprepared runways. ${ }^{9}$ As part of the SACD program sponsored by the AFRL, an aircraft math model of the SACD and corresponding baseline flight control system was developed and delivered to NASA. For this PIO control research evaluation, NASA augmented the baseline flight control system with a research control allocation system, the Control Allocation technique to recover from Pilot Induced Oscillations (CAPIO) allocator. More information on the CAPIO allocator can be found in Ref. 10 and Ref. 11 .

Four configurations of the research aircraft and flight control system were flown. The factors that differentiated the four configurations were the control allocator used and the rate limiting imposed on the control surfaces. To recognize the impact the CAPIO system had on system characteristics, two versions of the control allocator were flown. The first version employed the baseline allocator and served as the reference for measuring performance. The second version employed the CAPIO allocator.

The control surface rate limits were set at a nominal value and a reduced value for each task. The aircraft serving as the nominal configuration for both tasks operated with maximum rates of $100^{\circ} \mathrm{s}$ for all actuators. The aircraft configurations with reduced actuator rates operated with limits of $40 \%$ s. The reduced actuator rates were chosen such that actuators would encounter their limits to accomplish the task. This would introduce phase lag that would induce PIO tendencies, thus providing sufficient differences in system characteristics between the nominal and reduced rate limited aircraft configurations.

\section{Experimental Procedure}

The simulation was conducted over the course of two weeks. During those two weeks, seven test pilots participated in the study. Orientations were held to brief each pilot on the experiment's background, objective, task, procedures and aircraft system. Pilots were allowed one one-hour warm-up session to become familiar with the task. The familiarization periods used a different sum-of-sines to drive the boundaries than the subsequent practice and data runs. After the pilots were familiar with the task and aircraft, they began the experiment. Prior to collecting data for each configuration, a series of practice runs was conducted until the pilots felt they were achieving consistent results. The pilots were required to fly a minimum of three practice and three data runs for each configuration. At the conclusion of each practice and data run, performance feedback was provided to the pilot via an end-of-run display in the cockpit. Subjective and objective data was collected throughout and upon the completion of the data runs, as described in the following subsection. Due to the demanding nature of the task, pilots were given a break after flying two or three configurations to reduce pilot fatigue.

\section{E. Collection of Objective and Subjective Data}

Both objective and subjective data were collected during the motion-based simulation evaluation. The objective data recorded digitally during the simulation evaluation was in three formats. These formats include the simulation time history data with performance standard data, end-of-run pilot displays, and video with audio recording. The subjective data collected for each configuration was in the form of PIO ratings and pilot comments. The PIO ratings were captured using the traditional PIO rating scale, which provides a numerical rating meant to reflect the pilot's perception of the aircraft's performance and flight characteristics for a given task. Verbal comments expressed by pilots were recorded and written comments were captured on pilot comment cards unique to the task. These comments provide insight into how each pilot viewed the task and perceived the system's overall performance.

\section{Results and Discussion}

A total of 216 runs of the BAT task were recorded during the course of the two-week experiment. After removing runs for the project pilot and practice runs, there were 121 runs available for data analysis across four configurations and seven pilots.

PIOs experienced during the runs were identified visually by analyzing plots of the simulation data. As described in Section II, there is a phase lag between the pilot's commands and the aircraft's response during a PIO. Since the BAT task in this simulation was designed to expose PIO tendencies in the longitudinal axis, the PIOs of interest corresponded to pilot's commands through the longitudinal stick position and the aircraft's response in pitch angle. A limited analysis showed that PIOs identified by the data signals for the longitudinal stick position and aircraft 
pitch angle also corresponded to oscillations and phase lag in the desired rotational accelerations determined by the flight control system and the assumed achieved rotational acceleration, which is the acceleration that would result from the control surface deflections commanded by the flight control system. These latter signals were reviewed to visually identify PIOs. The system was declared to be in a PIO when two criteria were met. First, three or more peaks must be observed in the assumed achieved acceleration signal, with the peak size greater than twenty percent of the maximum amplitude. Second, there must be a visibly significant phase lag between the desired acceleration and the following assumed achieved acceleration signals. For each PIO identified, the number of peaks and their duration were measured started at the second of the consecutive peaks.

\section{A. Task Performance}

The criteria used to measure the performance during the BAT task was the length of time spent in flight before the bore-sight encountered the box's boundary. It was assumed that aircraft-pilot systems that are less PIO prone would fly the task for a longer time before failure. This assumption was checked by comparing the number of peaks/minute experienced during PIOs with the failure time. The result shown in Figure 5 indicates that while reduced PIO activity is a necessary condition for longer BAT task times, it does not guarantee better performance in time to failure.

Failure times shown in Figure 5 also appear to be grouped. To review where in the sum-of-sines pattern failures occurred, the performance data is compiled together for the 30 -second window of time spanning the pattern by overlaying the results from 1 second to 31 seconds with data from 31 to 61 seconds, 61 to 91 seconds, and 91 to 121 seconds. The distribution of failures along the BAT task pattern is shown in Figure 6. The largest group of failures at 30 seconds corresponds to when the boundaries jump closer together, which caused pilots with their bore-sight near the boundaries before the shift to fail as soon as the boundaries moved. The second and third largest groups of failures were around nine seconds and twenty seconds into the pattern occur at the time when both boundaries are moving very quickly, as seen in Figure 3, which would cause pilots to fail if they were not at or near full stick deflection.

\section{B. PIO Generation}

The intention of the experiment design was to reliably create PIOs in a controlled, repeatable manner. Results from the simulation evaluation confirm that the BAT task was successful in creating PIOs. Results also reinforce the theory that PIOs are due to a combination of the aircraft, the pilot, and the trigger.

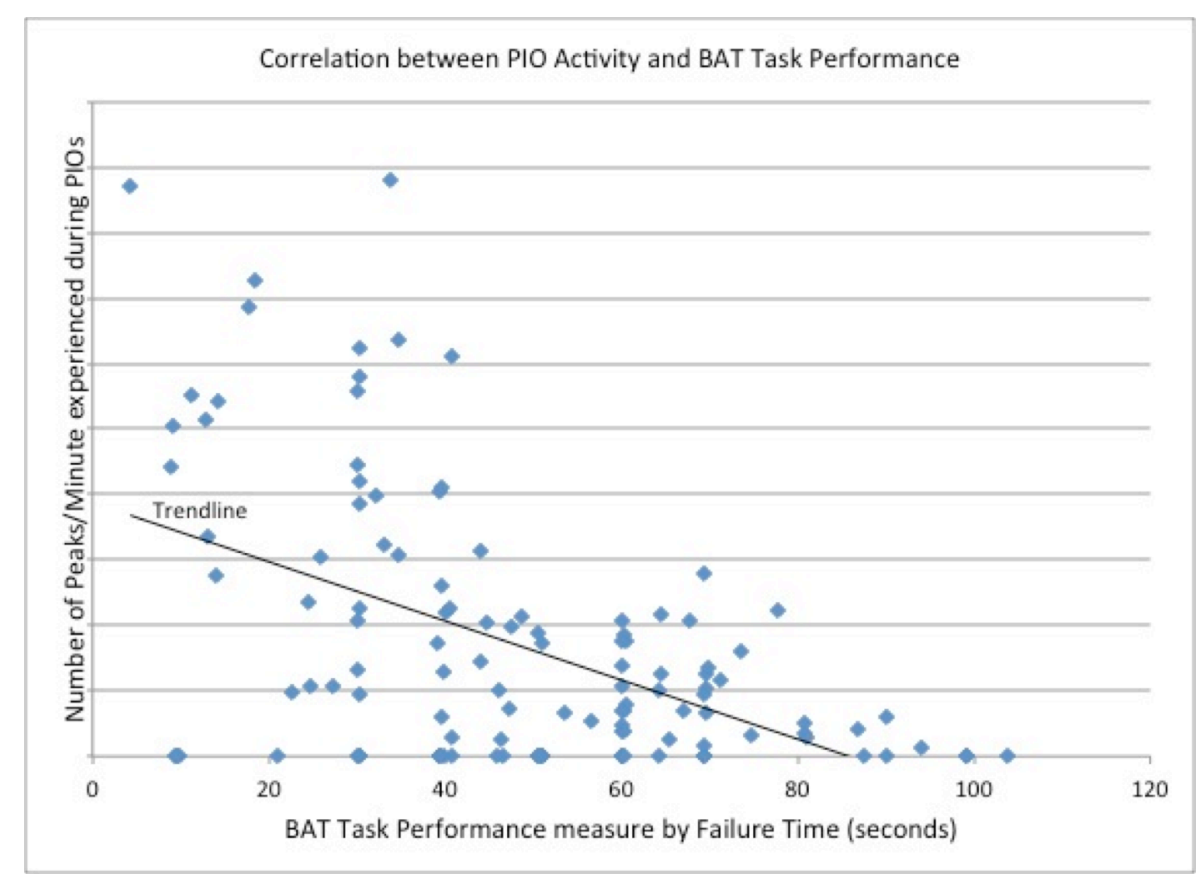

Figure 5. Correlation between PIO Activity, in Peaks per Minute, and BAT Task Performance, in failure time. 


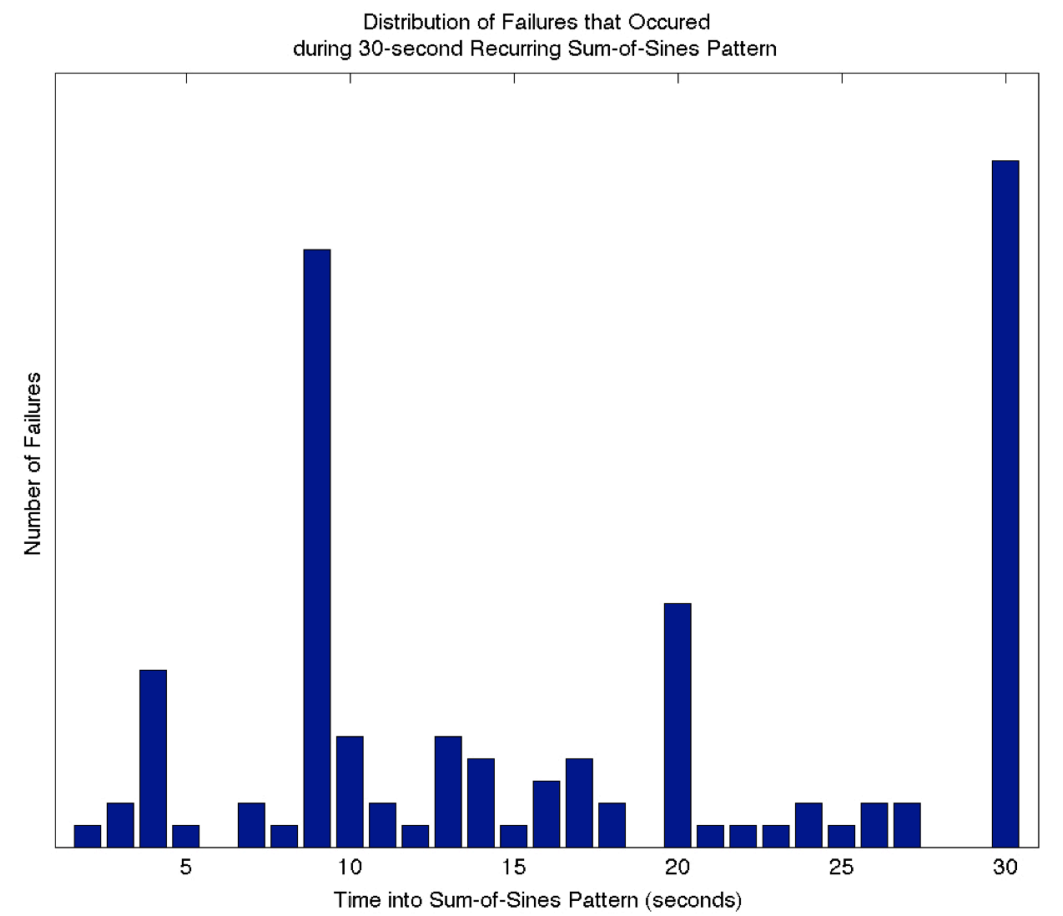

Figure 6. Distribution of Failures that Occurred during Recurring Sum-of-Sines Pattern.

Table 3. PIO Generation Data by Configuration.

\begin{tabular}{|l|c|c|}
\hline Configuration & Total \# of Runs & \% of Runs with PIO \\
\hline All & 121 & 72 \\
\hline Baseline Controller, $100^{\circ}$ /s Rate Limit & 26 & 42 \\
\hline CAPIO Controller, $100^{\circ}$ s Rate Limit & 23 & 43 \\
\hline Baseline Controller, 40 /s Rate Limit & 34 & 91 \\
\hline CAPIO Controller, 40 \% Rate Limit & 38 & 92 \\
\hline
\end{tabular}

Table 4. PIO Generation Data by Pilot.

\begin{tabular}{|c|c|c|c|c|}
\hline \multirow[b]{2}{*}{ Pilot } & \multirow{2}{*}{$\begin{array}{c}\text { Total \# of Runs } \\
\text { (All Configurations) }\end{array}$} & \multicolumn{3}{|c|}{$\%$ of Runs with PIO } \\
\hline & & $\begin{array}{c}\text { All } \\
\text { Configurations }\end{array}$ & $\begin{array}{c}\text { Configurations with } \\
100^{\circ} \% \text { s Rate Limit }\end{array}$ & $\begin{array}{c}\text { Configurations with } \\
40^{\circ} / \mathrm{s} \text { Rate Limit }\end{array}$ \\
\hline 1 & 19 & 58 & 22 & 90 \\
\hline 2 & 14 & 50 & 14 & 86 \\
\hline 3 & 16 & 69 & 29 & 100 \\
\hline 4 & 14 & 86 & 75 & 100 \\
\hline 5 & 19 & 53 & 0 & 77 \\
\hline 6 & 19 & 100 & 100 & 100 \\
\hline 7 & 20 & 85 & 67 & 93 \\
\hline
\end{tabular}

The aircraft configuration played a major role in the system's susceptibility to PIOs. In particular, the actuator rate limit influenced the instances of PIOs. Table 3 shows the percentage of runs that had at least one PIO, broken down by configurations. Configurations with a nominal actuator rate limit of $100^{\circ}$ s experienced at least one PIO 
during approximately $42 \%$ of runs, while configurations with a reduced actuator rate limit of $40 \%$ s experienced at least one PIO during approximately $91 \%$ of runs. The demanding task in combination with the reduced actuator rate limits lead to the system being more prone to Category II PIOs.

Piloting style also influenced the generation of PIOs during the BAT task. Table 4 shows the percentage of runs with at least one PIO, broken down by pilot for all configurations and for groupings of configurations by rate limit. Since each pilot operates at a their own unique gain, the percentage of runs with PIOs for all configurations ranged broadly from $50 \%$ to $100 \%$.

\section{Pilot's Perspective}

While the BAT task was successful in accomplishing the experiment objectives, pilot opinions of the BAT task were generally negative. The difficulty of the task, which required intently focusing on moving lines for extended periods of time, appeared to both strain the eyes, and be mentally exhausting.

\section{Recommendations for Future Implementation of BAT Task in Simulation}

To increase pilot receptivity of the BAT task, and ensure the ability to reliably create PIOs in a controlled, repeatable manner, the following recommendations are made for future implementations of the BAT task in simulation.

- Adjust the motion of the boundaries to have the longitudinal boundaries continuously move closer rather than jumping closer at predetermined time increments. This would eliminate the large group of failures that occur when the boundaries jump.

- Remove the lateral boundaries of the box, leaving only the longitudinal boundaries. At times, the lateral boundaries became an unnecessary distraction.

- Add a point-tracking target in the center of the boundaries. This would allow pilots to focus at the center of the task and to establish a mid-point between the boundaries. The idea of combining pointtracking and boundary avoidance tracking actually dates back to the original conception of the BAT task in Gray's 2004 paper.

- Tune a major frequency of the sum-of-sine's pattern to be at or near the aircraft's crossover frequency. If done correctly, this may incite even more, possibly divergent PIOs.

\section{Conclusion}

The boundary avoidance tracking task, as implemented in the simulation experiment described, was successful in creating PIO events in $72 \%$ of data runs. The task was a useful tool for researchers developing technology to recover from PIO because the task enabled the regular creation of PIOs in a controlled setting. Since a PIO is caused by a combination of aircraft, pilot, and trigger, the BAT task alone is not sufficient to regularly create PIO events; for maximum affect a PIO-prone aircraft and high gain pilot are necessary.

\section{Acknowledgments}

The authors extend their appreciation to two projects within the NASA Aeronautics Research Mission Directorate - the Fundamental Aeronautics Program's Subsonic Fixed Wing Project and the Integrated Systems Research Program's Environmentally Responsible Aviation Project - for their support of this research.

\section{References}

MIL-HDBK-1797, Department of Defense Handbook, "Flying Qualities of Piloted Aircraft,” December 1997

McRuer, D., "Pilot-Induced Oscillations and Human Dynamic Behavior," NASA Contractor Report 4683, July 1995

"Flight Control Design Best Practices," Research and Technology Organization, North Atlantic Treaty Organization, Neuilly-sur-Seine Cedex, France, December 2000, RTO-RT-29

4 Gray, W., "Boundary - Escape Tracking: A New Conception of Hazardous PIO," USAF Test Pilot School, Edwards AFB, CA, September 2004

5 Curry, M., "STS Approach and Landing Test, Pilot-Induced Oscillation (PIO)," Movie Collection Dryden Flight Research Center, URL: http://www.dfrc.nasa.gov/gallery/movie/STS/HTML/EM-0084-02.html [cited 4 October 2011]

6 Dotter, J. D., "An Analysis of Aircraft Handling Quality Data Obtained from Boundary Avoidance Tracking Flight Test Techniques," Air Force Institute of Technology, Wright-Patterson Air Force Base, Ohio, March 2007 
7 Warren, R. D., "An Investigation of the Effects of Boundary Avoidance on Pilot Tracking," Air Force Institute of Technology, Wright-Patterson Air Force Base, Ohio, September 2006

8 Blake, Ryan D. "Boundary Avoidance Tracking: Consequences (and Uses) of Imposed Boundaries on Pilot-Aircraft Performance," Thesis. Air Force Institute of Technology, 2009.

9 Broad Agency Announcement by the Air Force Research Laboratory, Air Vehicles Directorate, Aeronautical Sciences Division, AFRL/VAAA, Speed Agile Concept Demonstration, Number 07-07-PKV

10 Craun, R. W., Acosta, D. M., Beard, S. D., Leonard, M. W., Hardy, G. H., Weinstein, M., Yildiz, Y., "Motion-Based Piloted Simulation Evaluation of a Control Allocation Technique to Recover from Pilot Induced Oscillations," AIAA Guidance, Navigation, and Control Conference, AIAA, Boston, Massachusetts, August 2013

11 Leonard, M. W., "Real-Time Optimization for use in a Control Allocation System to Recover from Pilot Induced Oscillations," AIAA Guidance, Navigation, and Control Conference, AIAA, Boston, Massachusetts, August 2013

12 Schroeder, J. and Chung, W., "Simulator Platform Motion Effects on Pilot-Induced Oscillation Prediction" Journal of Guidance, Control, and Dynamics, Vol. 23, May-June 2000

13 Aponso, B., Beard,S., Schroeder, J., "The NASA Ames Vertical Motion Simulator - A Facility Engineered for Realism," Royal Aeronautical Society Spring 2009 Flight Simulation Conference, London, UK, 3-4 June 2009

14 Mitchell, D. G., and Hoh, R. H., "Development of Methods and Devices to Predict and Prevent Pilot Induced Oscillations," SBIR Phase II Report, AFRL-VA-WP-TR-2000-3046, December 2000 